\title{
Role of the BCA2 Ubiquitin E3 Ligase in Hormone Responsive Breast Cancer
}

\author{
Angelika M. Burger ${ }^{1}$, Fathima Kona ${ }^{1}$, Yutaka Amemiya ${ }^{2}$, Yuguang $\mathrm{Gao}^{2}$, Stephanie Bacopulos ${ }^{2}$ and \\ Arun K. Seth ${ }^{*}, 2$ \\ ${ }^{I}$ Barbara Ann Karmanos Cancer Institute and Department of Pharmacology, Wayne State University School of \\ Medicine, Detroit, MI, USA \\ ${ }^{2}$ Sunnybrook Research Institute and University of Toronto Department of Laboratory Medicine and Pathobiology, \\ Toronto, ON, Canada
}

\begin{abstract}
The BCA2 protein contains a RING H2 finger and a Zn finger near the N-terminus and has E3 ligase activity. RING finger proteins play critical roles in mediating the transfer of ubiquitin and ubiquitin like modifiers to heterologous substrates as well as to the RING finger proteins themselves. Protein modification by ubiquitin and small ubiquitin-related modifier (SUMO) plays a pivotal role in protein homeostasis and is critical to regulating basic cellular processes such as proliferation, differentiation, apoptosis, intracellular signaling, and gene-transcriptional regulation. The addition of ubiquitin or SUMO can modulate the ability of proteins to interact with their partners, alter their patterns of sub-cellular localization and control their stability. It is clear that SUMO influences many different biological processes however recent data suggest that it is specifically important in the regulation of transcription. BCA2 is an E3 ligase that interacts with the SUMO conjugating enzyme Ubc9. It could therefore function as an E3 in the sumoylation of various transcription factors. We have found that the BCA2 is co-expressed with the estrogen receptor in $74 \%$ of ER-positive invasive ductal carcinomas from a 635 member breast cancer cohort $(\mathrm{p}=0.004)$. At the cellular level, BCA2 co-localizes with ER and it appears that at the transcriptional level BCA2 mRNA expression is regulated by estrogen. Bioinformatic analysis of the BCA2 promoter region revealed ER and PR binding sites as well as that of other more general transcription factors. The data presented here provides an overview of the potential involvement of the BCA2 in hormone responsive breast cancer and opens up avenues that should be exploited to better understand the regulation of ER expression, growth of breast cancer cells, and the importance of BCA2.
\end{abstract}

Keywords: BCA2, SUMO, hormone-responsive, breast cancer, transcription.

\section{INTRODUCTION}

At the cellular level, transcription factors are tightly controlled by their rates of synthesis and degradation. Many transcription factors are maintained at an appropriate level by targeted addition of polyubiquitin chains and subsequent degradation in the proteasome [1]. While polyubiquitination targets proteins for degradation, monoubiquitination or their modification by small ubiquitin-like modifiers such as SUMO, alters subcellular localization and can change their activity [1]. Important transcription factors known to be regulated by ubiquitination or sumoylation are HIF1- $\alpha$, cMyb, c-Jun, Oct4, ETS1, and the ER among others [2-4]. Each of these transcription factors regulates the expression of a large number of target genes. Alterations of these transcription factors are frequently involved in tumorigenesis $[1,5,6]$.

In response to circulating estrogen, the estrogen receptor (ER) regulates the genetic programs of cell cycle progression and growth in normal mammary gland and breast cancer

*Address correspondence to this author at the Sunnybrook Health Sciences Centre, 2075 Bayview Avenue, Toronto, Ontario, M4N 3M5, Canada; Tel: 416 480-6100X7897; Fax: 416480 5703; E-mail: arun.seth@utoronto.ca epithelial cells. This critical transcription factor has two receptor forms, ER $\alpha$ and ER $\beta$. ER $\beta$ demonstrates lower hormone-dependent transcriptional activity [7]; therefore $E R \alpha$ is considered the primary receptor for mammary gland development and function [8]. However, little is known of how the stability and expression of ER is regulated. Recent studies indicate that the ER is monoubiquitinated and sumoylated when interacting with BRCA1, which might lead to repression of ER transcriptional activation $[9,10]$. Furthermore, cancer-predisposing mutations in BRCA1 were observed to abrogate ER ubiquitination [9], implicating ubiquitin E3 ligases as playing a major role in ER regulation and hormone responsive breast cancer.

We had previously identified Breast $\underline{\text { Cancer }} \underline{\text { Associated }}$ gene 2, BCA2 (synonymous with T3A12/ZNF364/Rabring7/ RNF115), a novel RING-finger ubiquitin E3 ligase, by subtractive hybridization cloning in breast carcinoma cell lines [11]. Subsequently we found that BCA2 is expressed in primary invasive breast cancers and is associated with a positive estrogen receptor status and outcome $[12,13]$. Here we describe the interaction of the BCA2 protein with the SUMO conjugating enzyme Ubc9, its regulation by ER, and its potential involvement in transcriptional regulation of hormone responsive breast cancers. 


\section{BCA2 and ER Co-Expression in Invasive Breast Cancer}

Estrogen regulates the proliferation and development of tissues expressing estrogen receptors and is a risk factor for breast cancer development. Ligand binding activates both ER $\alpha$-dependent transcription and ER $\alpha$ ubiquitination [14]. $\mathrm{ER} \alpha$ ubiquitination and proteasome activity are intimately linked to ER $\alpha$-dependent transcriptional activation $[14,15]$. Proteasome inhibitors and mutations that inhibit co-activator binding both abrogate ligand-mediated ER $\alpha$ proteolysis and ERE transcriptional activity [15]. Different ligands stimulate $\mathrm{ER} \alpha$ proteolysis to different degrees [16], and the ubiquitin ligases BRCA1 [17], MDM2 [18], and E6AP [19] can all stimulate estrogen-induced transcriptional activity.

The BCA2 protein is an ubiquitin ligase co-expressed with the ER in breast cancers [12]. We have studied BCA2 protein expression in a large collection of over 1,000 invasive mammary carcinomas, termed the Henrietta Banting Breast Cancer Collection (HBBCC) [12,13]. This breast cancer tissue resource located at Sunnybrook Health Sciences Centre, Toronto, has been well characterized for established molecular markers such as ER, PR (progesterone

A

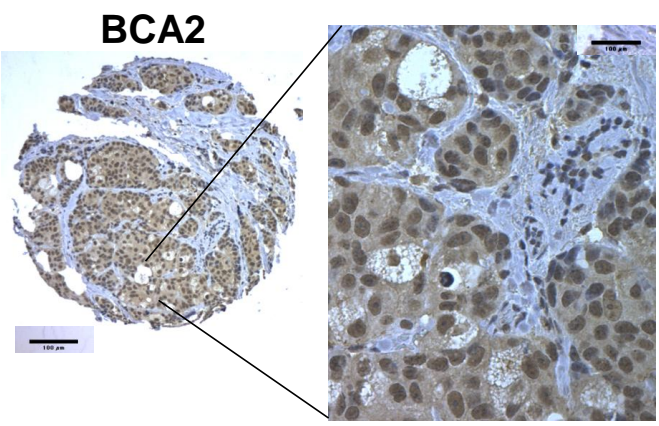

B BCA2
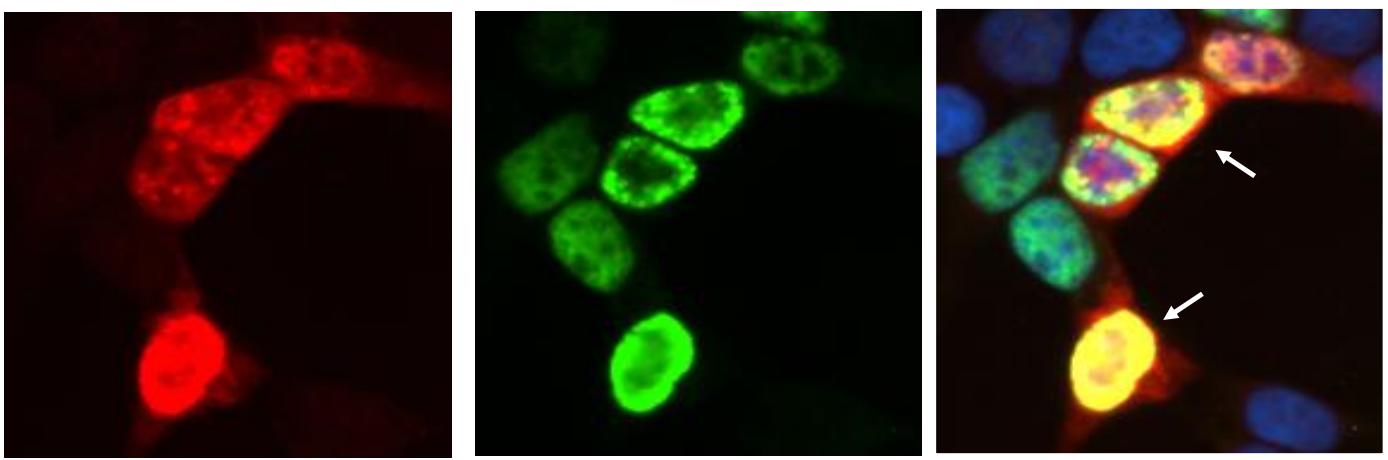

Fig. (1). (A) Comparison of BCA2 and ER expression by immunoperoxidase staining in a representative case of invasive breast cancer from the HBBCC tissue microarrays (method described in detail in [12]). Consecutive tissue microarray sections were stained for BCA2 and ER and viewed at $4 \mathrm{X}$ magnification, size bar $=100 \mu \mathrm{m}$. Enlargements at 40x magnification were captured from the $4 \mathrm{X}$ core areas as outlined by a box. The data reveal co-expression of ER and BCA2 in the nuclei of cells staining positive for the antigens (brown color: diaminobenzidine). Overall, 67\% of the 945 invasive HBBCC cases were positive for ER and of those 74\% were positive for both, ER and BCA2 [12]. (B) Colocalization of ER (green, FITC-labeled anti-mouse antibody) and BCA2 (red, TRITC-labeled anti-rabbit antibody) in the nuclei (merge: yellow, white arrows; nuclei were counterstained with DAPI $=$ blue) of HEK293T cells. $5 \mu \mathrm{g}$ of a wild type estrogen receptor vector construct [43] was co-transfected with $5 \mu \mathrm{g}$ wild type BCA2 in pCMV-tag2B (FLAG tag) into HEK293T cells and after 48 hrs fixed in methanol:acetone $(1: 1)$ and stained with monoclonal anti-estrogen receptor $\alpha$ antibody clone $6 \mathrm{~F} 11$ (Invitrogen, 08-1214) and a rabbit antiFLAG antibody (Sigma, St. Louis, MO) following procedures as described previously [12,44]. A Leica DM5500 microscope and Improvision software with a Retiga camera were used to capture and merge fluorescence signals. Size bar $=15 \mu \mathrm{m}$. 
Table 1. Summary of BCA2 and ER Expression Pattern in Invasive Breast Cancers

\begin{tabular}{|c|c|c|c|c|c|c|}
\hline & \multicolumn{2}{|c|}{ Total Cases } & \multicolumn{2}{|c|}{ LN Positive } & \multicolumn{2}{|c|}{ Regional Recurrence } \\
\hline & $\mathbf{n}$ & $\%$ & $\mathbf{n}$ & $\%$ & $\mathbf{n}$ & $\%$ \\
\hline $\mathrm{BCA} 2 \leq 1$ & 415 & 43.9 & 213 & 51.3 & 35 & 8.6 \\
\hline BCA2 >1 & 530 & 56.1 & 229 & 42.2 & 28 & 5.3 \\
\hline $\mathrm{ER}+$ & 635 & 67.1 & ND & & ND & \\
\hline ER- & 310 & 32.9 & ND & & ND & \\
\hline $\mathrm{ER}+/ \mathrm{BCA} 2>1$ & 392 & 74.0 & ND & & ND & \\
\hline
\end{tabular}

$\mathrm{ND}$, not determined; $\mathrm{n}$, number; $\%$, percent.

ER+, estrogen receptor positive; ER-, estrogen receptor negative.

213), were more likely to have lymph node metastasis at presentation than those with higher BCA2 levels $(49.9 \%$ of $229, \mathrm{p}=0.02)$. Second, women with invasive breast cancers with low BCA2 (8.6 \% of 415) were more likely to experience regional re-occurrence than those with higher BCA2 $(5.3 \%$ of 530$)(p=0.05)$ (Table 1). The third significant correlation was very strong: nuclear BCA2 expression was correlated with a positive estrogen receptor status (Fig. 1A). $67.1 \%$ of all 945 invasive HBBCC cases stained positive for ER expression. $74 \%$ of breast cancers that were ER-positive had also nuclear BCA2 $>1(p=0.004)$ [12]. Because of the significant statistical correlation found between invasive breast cancers expressing ER and BCA2, we compared the pattern of expression of these two proteins. Core by core analysis of consecutive tissue sections indicated that BCA2 and ER are mostly co-expressed (odds ratio $=1.5$ ) (Fig. 1A), cells strongly positive for BCA2 were positive for ER. It is important to note that high expression of BCA2 is not beneficial at the cellular level for inhibiting cancer progression and may be a contributing factor in metastasis. However the high correlation of co-expression with ER makes higher expression of BCA2 a marker for good prognosis in breast cancer due to the availability of highly effective treatment options.

\section{BCA2 may be Transcriptionally Regulated by the Estrogen Receptor}

$\mathrm{ER} \alpha$ is a $66-\mathrm{kDa}$ nuclear hormone receptor transcription factor [20]. Upon ligand binding, ER $\alpha$ dimerizes and associates with co-activators and chromatin remodeling factors to activate transcription of genes containing estrogen response elements [21]. The ER $\alpha$ phosphorylation state affects coactivator binding and ER $\alpha$-DNA binding affinity [22].

BCA2 is expressed in $74 \%$ of ER positive invasive breast cancers. Co-localization of BCA2 and ER protein can also be demonstrated in cell lines that express neither endogenous
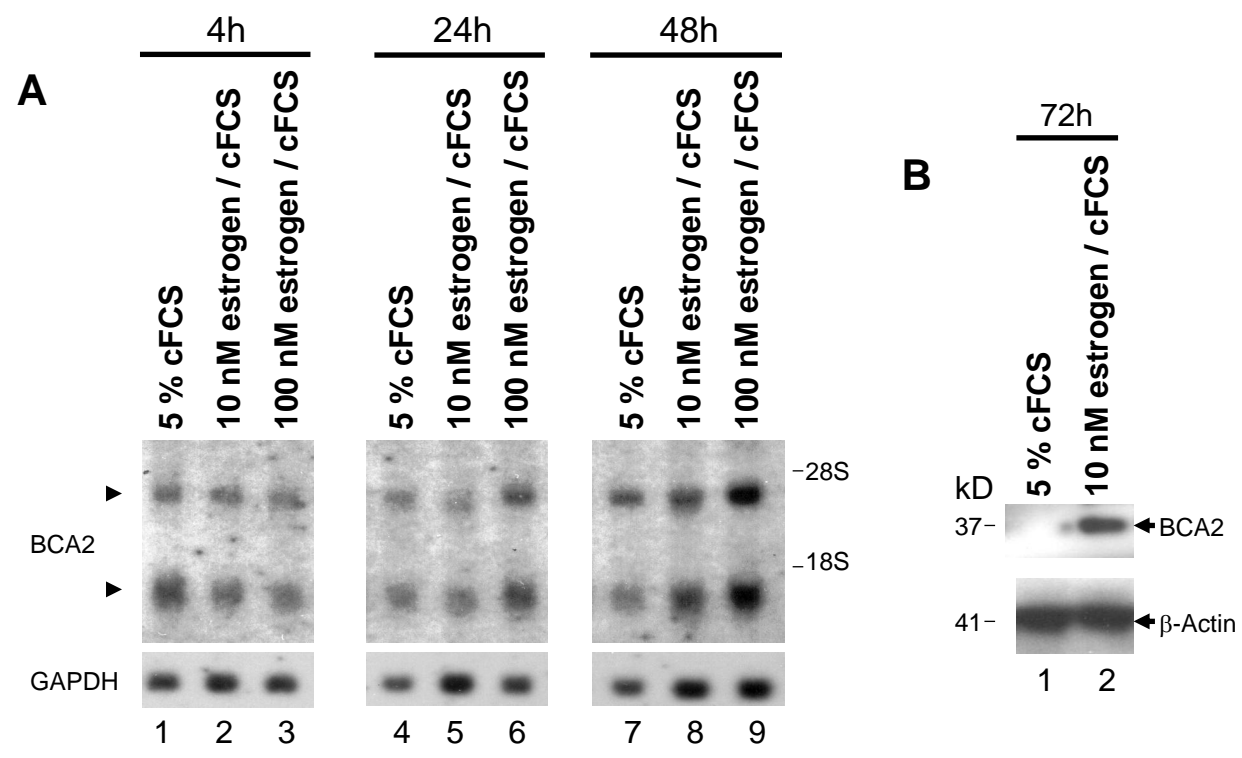

Fig. (2). (A) Northern blot probed with BCA2 cDNA showing BCA2 expression in RNA extracted from ER-positive T47D cells treated with either $5 \%$ cFCS (charcoal-stripped fetal calf serum), or cFCS treated with $10 \mathrm{nM}$ or $100 \mathrm{nM}$ of estrogen, over a time-course of $48 \mathrm{hrs}$. The same membrane was re-probed for the GAPDH housekeeping gene as loading control. RNA extraction and Northern blots were performed as reported by us before [11,12]. A time and concentration dependent response of BCA2 mRNA to estrogen is seen with the most prominent effects observed at $48 \mathrm{hrs}$ in estrogen-supplemented cFCS. (B) Western blot probed with anti-BCA2 antibodies showing BCA2 protein expression in whole cell lysates from T47D cells that were grown in 5\% cFCS or cFCS with 10nM estrogen after 72 hours [12]. Beta-actin was as a loading control. 

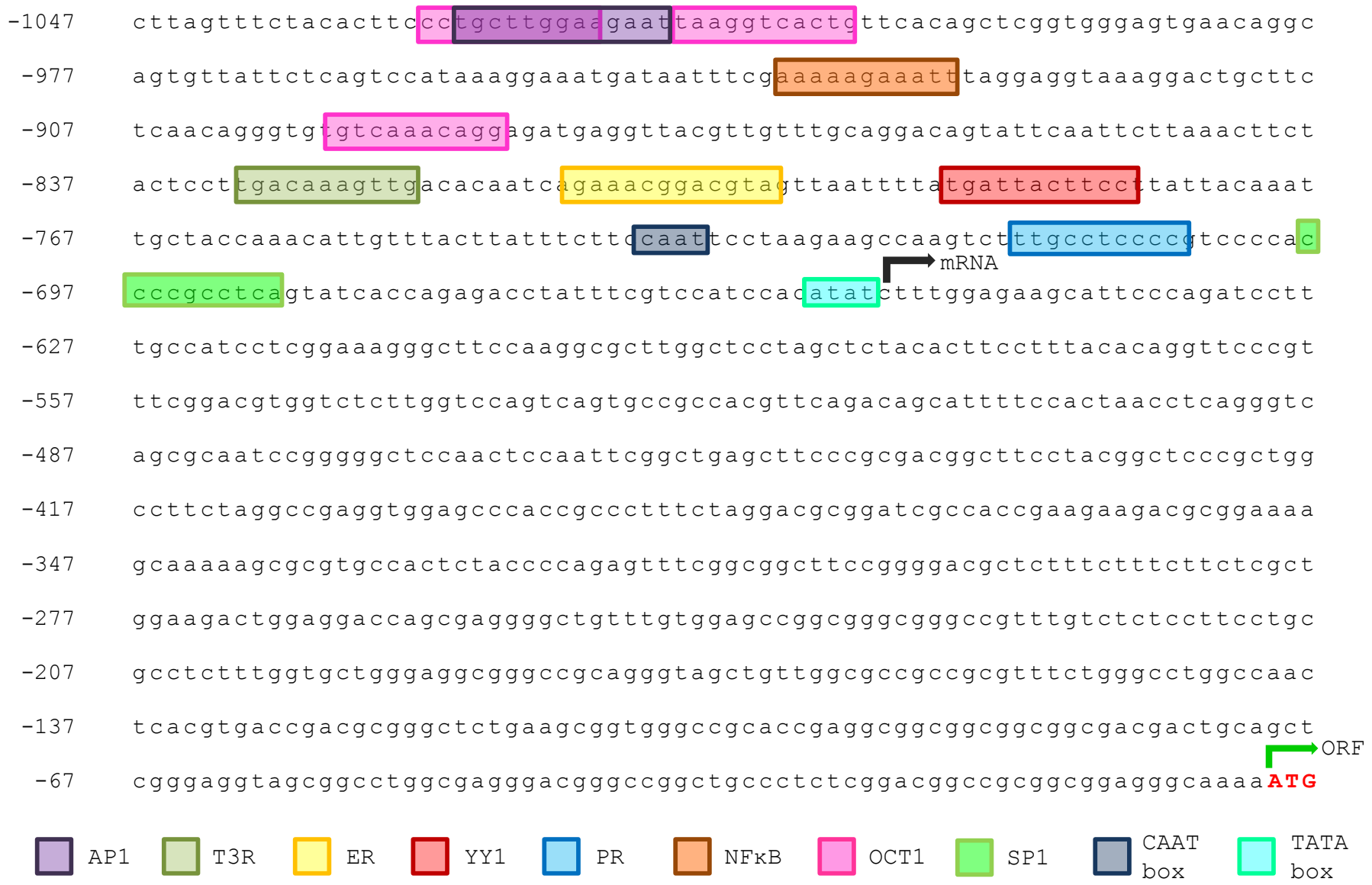

TATA box

Fig. (3) BCA2 promoter. Annotation of transcription factor binding sites (indicated as colored boxes) from bioinformatics analysis of the BCA2 promoter, accession NT_030171.4|Hs19_30426. BCA2 transcriptional targets and their position in the promoter region are also listed in (Table 2).

BCA2 nor ER (Fig. 1B). The nuclear co-localization of BCA2 and ER indicates that BCA2 could function as an E3 ligase for ER and regulate its stability as shown for other RING domain ubiquitin ligases.

BCA2 may also be an estrogen (17-beta-estradiol) responsive gene. T47D cells are estrogen responsive when grown in charcoal-stripped fetal calf serum (cFCS) [23]. The ER-positive, BCA2-positive T47D human breast cancer cell line was used to study estrogen effects on BCA2 RNA and protein expression in cells that were grown in medium cFCS, which is growth factor deprived (Fig. 2). For the experiment depicted in (Fig. 2A and B), T47D cells were grown in cFCS for an extended period of time (72 hrs.), and further cultured in RPMI 1640 medium supplemented with $5 \%$ cFCS or $5 \%$ cFCS that contained 10 or $100 \mathrm{nM}$ estrogen, for $4,24,48$ and 72 hours. Northern blotting results show that when cells were grown in $5 \%$ cFCS transcription of BCA2 mRNA is similar over a time course of 4-48 hours. Cells treated with estrogen showed increased induction of BCA2 mRNA after 48 hours. Moreover, the induction of BCA2 was concentration dependent; $100 \mathrm{nM}$ estrogen strongly induced BCA2 after 24 and $48 \mathrm{hrs}$, whereas $10 \mathrm{nM}$ estrogen induced BCA2 to $50 \%$ of the levels seen for $100 \mathrm{nM}$ estrogen (Fig. 2A). Induction of BCA2 by estrogen at the RNA levels translated into expression of BCA2 in T47D cells at the protein level.
Protein levels are shown after 72 hours in $5 \% \mathrm{cFCS}$ or cFCS supplemented with $10 \mathrm{nM}$ estrogen (Fig. 2B) indicating that estrogen induces BCA2 RNA and protein in cFCS-cultured T47D cells and that BCA2 is an estrogen responsive gene.

We searched for transcription factor binding sites in the BCA2 promoter region (Fig. 3) using a bioinformatics approach. To predict the potential transcription factors binding to the BCA2 promoter region, an 1100 bp sequence upstream of exon 1 was analyzed. We preformed in silico analysis of the genomic region from $1.1 \mathrm{~kb}$ upstream and $235 \mathrm{bp}$ downstream $(-1101$ to +235$)$, with the Alibaba 2.1 (www.generegulation.com) software using the TRANSFAC transcription factor binding sites collection. This analysis revealed a canonical CAAT box (-737 to -734) and reverse TATA box (-656 to -653) and several putative binding sites for general transcription factors; nuclear hormone receptors were also identified and are listed in (Table 2) and (Fig. 3).

Up-stream of the TATA box we found a half site for ER, multiple sites for Oct-1, and SP1 (Table 2). Other transcriptional factor binding sites include AP-1, NF- $\kappa$ B, T3R, YY1, and PR (Table 2, Fig. 3). SP1, AP-1, Oct-1 and YY1 are ubiquitous transcription factors that have a fundamental role in normal biologic processes including differentiation, replication, cellular proliferation and apoptosis [24-27]. 
Table 2. Potential Regulatory Element Binding Sites in the BCA2 Promoter Region

\begin{tabular}{|c|c|c|}
\hline Regulatory Element Binding Sites & Start & Stop \\
\hline \hline AP1 & -1027 & -1018 \\
\hline NF-kappa B & -939 & -930 \\
\hline T3R & -828 & -819 \\
\hline ER & -811 & -800 \\
\hline YY1 & -790 & -780 \\
\hline PR & -716 & -707 \\
\hline SP1 & -698 & -689 \\
\hline OCT1 & -1029 & -1020 \\
& -1017 & -1008 \\
& -894 & -885 \\
\hline TATA Box & -656 & -653 \\
\hline CAAT Box & -737 & -734 \\
\hline
\end{tabular}

AP1: Activator Protein 1, NF-kappa B: Nuclear factor kappa-light-chain-enhancer of activated B cells, T3R: Triiodothyronine Receptor, ER: Estrogen Receptor, YY1: Ying Yang 1, PR: Progesteron Receptor, OCT1: Octamer-binding transcription factor 2, SP1: Specificity Protein 1

T3R and NF- $\mathrm{BB}$ have more defined functions in cells [28-30]. Thyroid hormone receptor (T3R) generally requires the binding of its high affinity ligand. However mutants of the ligand-binding domain have been implicated in inter- actions with other proteins, suggesting participation of other transcription or accessory factors in the hormone-independent activity of T3R.

The estrogen receptor and progesterone receptor (PR) receptor binding sites are most noteworthy in view of the induction of BCA2 RNA and protein expression seen in response to estrogen (Fig. 2).

\section{Sumoylation and Transcriptional Activation of ER and its Target Genes}

Sumoylation is a dynamic process and its outcomes are extremely diverse, ranging from changes in localization to altered activity and stability of some of the modified proteins. [31]. Ubc9, a SUMO-conjugating enzyme, is essential for the sumoylation pathway as currently understood [32]. The number of known E3 ligases for SUMO modification is relatively low compared to the large number of known sumoylated targets, suggesting that many ligases await identification [33]. Our data presented in (Fig. 4) suggest that BCA2 may be involved in the conjugation of SUMO to targets such as ER and other transcription factors.

Sumoylation has been implicated in regulation of transcriptional activity either by modification of factors which induce or repress the transcription of genes, or through modification of histones [31]. Sumoylation of histones results in the recruitment of factors which promote heterochromatin formation, decreasing the amount of active gene transcription.

A

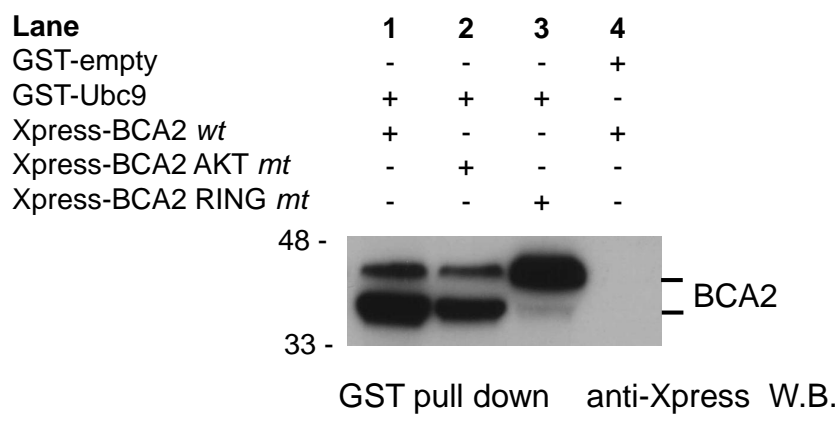

B

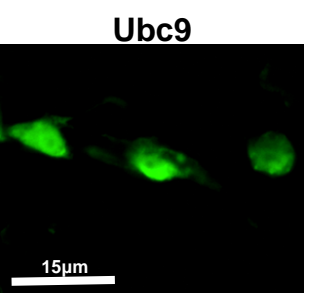

BCA2

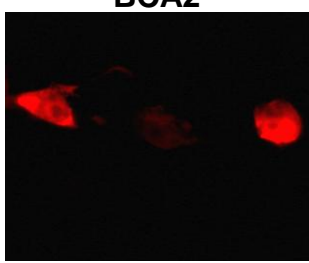

BCA2 \& Ubc9
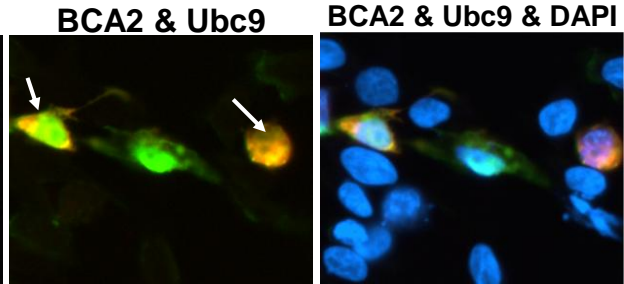

Fig. (4). (A) Bacterially expressed His/Xpress-tag vectors of BCA2 and GST vectors of Ubc9 were used for GST-pulldown assay as described in [37] and indicated to the left, lanes 1-4. Pull-down assays were performed from bacteriall cell lysates with GST-beads and Western blots (W.B.) probed with anti-Xpress tag antibodies detecting BCA2 and its mutants. (B) Co-localization of Ubc9 and BCA2 in HEK293T cells. Ubc9 was cloned into pCMV-tag2B and BCA2 into a GST expression vector as described in [12] and [39]. Cells were cotransfected and processed for immunofluorescence staining as outlined in figure legend 1B. Ubc 9 expression was detected by using FITClabeled (green, against monoclonal anti-FLAG antibodies) anti-mouse antibodies and BCA2 by using TRITC-labeled (red, against our polyclonal BCA2 antibody) anti-rabbit antibodies. Co-expression of Ubc9 and BCA2 (yellow) is indicated by white arrows. Co-localization is seen in both the nucleus and the cytoplasm. Nuclei are counterstained with DAPI (blue). Size bar $=15 \mu \mathrm{m}$ 


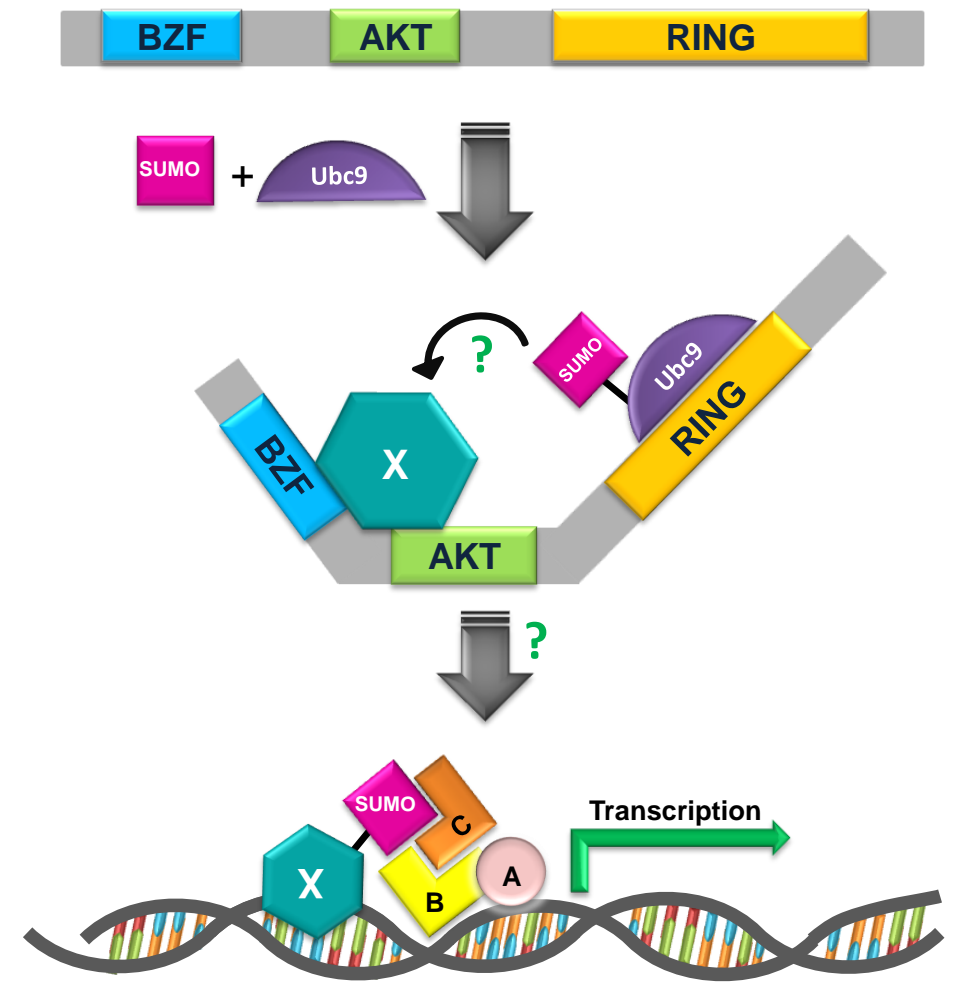

Fig. (5). Proposed model for BCA2 involvement in sumoylation. BCA2 with its known domains: BZF, $\underline{B} C A 2$ zinc finger domain; AKT, BCA2 AKT phosphorylation site; RING-finger domain, catalytic E3 ligase and probable E2 binding site. Ubc9, the SUMO-conjugating enzyme for the sumoylation pathway binds BCA2 likely in the RING domain, similar to the binding ubiquitin-conjugated E2s. This brings SUMO-conjugated Ubc9 into close proximity with the substrate (indicated by X) and allows the transfer of SUMO. Following sumolyation, the substrate may act as a factor in gene transcription though protein-protein interaction (with A, B, C) or DNA binding.

In the context of hormone-receptors, androgen receptor (AR), ER and PR, all have SUMO-conjugation sites; however the consequence of modification differs between the different receptors. ER is predominantly activated upon sumoylation. In contrast, when PR and AR are sumoylated, an inhibitory effect is observed [34].

ER sumoylation occurs strictly in the presence of estradiol and it appears that SUMO-1 regulates ER-dependent transcription (Figs. 4, 5) [10]. The SUMO E3 ligases PIAS1 and PIAS3, as well as Ubc9 were found to modulate ERdependent transcription independently from their SUMO-1 conjugation activity and provide a link between the SUMO and estrogen pathways [10].

Several of the transcription factors that bind in the BCA2 promoter region (Fig. 3, Table 1) have been shown to be modified by sumoylation including YY1, SP1 and IкB [32,35-37].

YY1 protein is sumoylated at lysine 288 as the major sumoylation site [35]. Many post-translational modifications have been implicated in the regulation of Sp1 activity including SUMO-1. SUMO- 1 is covalently conjugated to Sp1 within the N-terminal negative regulatory domain of Sp1. Compared with Sp1, sumoylation-deficient Sp1 mutants exhibit enhanced cleavage, increasing transcriptional activation. This is in contrast to the constitutively SUMO-1modified Sp1, which is deficient in proteolytic processing and is associated with inhibition of $\mathrm{Sp} 1$ transcriptional activity [36].

The NF- $\kappa \mathrm{B}$ inhibitory protein $\mathrm{I} \kappa \mathrm{B}$ is modified by SUMO-1 on lysine 21, which is also used for ubiquitin conjugation. Importantly, SUMO-1-modified I $\mathrm{KB}$ cannot be ubiquitinated and is resistant to proteasome mediated degradation, increasing the stability of the protein. As a result, signal-induced activation of NF- $\mathrm{KB}$ dependent transcription is inhibited [32]. While Ubc9 is the SUMO conjugating enzyme of I $\mathrm{I} B$, the cooperating E3 ligase remains unknown.

\section{BCA2 Interacts with the SUMO-Conjugating Enzyme UBC9}

RING-E3 ligases are major players in post-translational modification and protein degradation. Known to be imbalanced in cancer, many E3s have emerged as mutated, lost or overexpressed in breast cancer. E3 ligases together with kinases make-up approximately $15 \%$ of all cancer genes, suggested to be major regulators in cell growth and death pathways [38]. More recently RING-E3 ligases have been known to play a role in the SUMO pathway, which is thought to be an antagonist of the ubiquitination pathway, by stabilizing proteins and sterically competing for ubiquitin modification sites [2].

To identify potential substrates for the BCA2 E3 ligase, we had previously performed bacterial and yeast-two hybrid screens [39, unpublished data]. Several binding partners 
were isolated, including, Rab7, ubiquitin, 14-3-3, and the SUMO conjugating enzyme Ubc9 (Table 2) [40]. Ubc9 was confirmed by pull down assays using GST-tagged Ubc9 expressed from the pGEX4T3 bacterial expression vector. BCA2 variations were constructed in pET100 vectors containing N-terminal 6xHis and Xpress tags. Purified bacterial recombinant wild-type BCA2, as well as RING (C228A, C231A) and AKT (S132A, S133A) BCA2 mutants, were incubated with GST-Ubc9 from bacterial lysates. Following incubation, mixtures were subjected to SDS-page and Western blotting. Membranes were probed with antiXpress antibody (Invitrogen). We found that Ubc9 binds to all BCA2 variants (Fig. 4A). Moreover, when we expressed both BCA2 and Ubc9 in HEK293T cells, we saw colocalization with BCA2 in the cytoplasm and the nucleus suggesting that Ubc9 mediated sumoylation might be important to its nuclear as well as cytoplasmic function (Fig. 4B).

\section{CONCLUSION}

Endocrine therapy for women with metastatic breast cancer is one of the options for systemic treatment in the battle against breast cancer. The "best" strategy depends in large part on the molecular biology of particular cancers, e.g., the expression of hormone receptors and HER2/neu.

Although, several agents that interfere with ER signaling are clinically available, the understanding of their mechanisms is more complex than expected. Tissue- and cellspecific estrogen mechanisms depend upon the formation of a wide variety of co-regulatory complexes as well as variable ER subtypes and extra-nuclear signaling events.

It is well known that post-translational modifications, such as sumoylation, affect breast cancer by altering important regulatory proteins, transcription factors, growth factors and oncoproteins [41]. Innumerate cellular pathways are impacted through sumoylation, affecting transcriptional activity, protein stability and protein sub-cellular localization. Examples of sumoylated oncogenes and tumor suppressor genes include Mdm2, c-Myb, Rb, ER, and p53, all of which undergo sumoylation $[41,42]$. Here we showed that BCA2 is upregulated by ER and that it in turn could mediate the sumoylation of ER and other proteins through its interaction with Ubc9.

Genomic and proteomic approaches are often used to determine targets differentially regulated by sumoylation. Such work has broadened our understanding of how sumoylation affects ER associated signaling molecules function and localization in breast cancer cells. An example of this is our work with BCA2, where we identified its interactions with the ER and SUMO conjugating enzyme Ubc9. Ubc9 is the only E2 currently known to conjugate SUMO to substrates. It is possible that BCA2 may act as an E3 and catalyze the addition of SUMO to substrates, or BCA2 may auto-sumoylate to regulate its own function, similar to the POU domain transcription factor OCT4 which has been shown to bind Ubc9 [2]. We believe the interplay of SUMO, $\mathrm{BCA} 2$, and ER in breast cancer warrants further examination for potential clinical interventions that may exploit their molecular characteristics.

\section{ACKNOWLEDGEMENTS}

This work was supported by Award Number R01CA127258 from the National Cancer Institute, Developmental Therapeutics Program and by the Canadian Breast Cancer Research Alliance special program grant on metastasis.

$\begin{array}{ll}\text { ABBREVIATIONS } \\ \text { BCA2 } & =\text { Breast cancer associated gene } 2 \\ \text { SUMO } & =\text { Activator Protein 1 } \\ \text { AP1 } & =\text { Nuclear factor kappa-light-chain-enhancer } \\ \text { NF-kappa B } & \text { of activated B cells } \\ \text { T3R } & =\text { Triiodothyronine Receptor } \\ \text { ER } & =\text { Estrogen Receptor } \\ \text { ERE } & \text { Estrogen responsive element } \\ \text { YY1 } & \text { Ying Yang 1 } \\ \text { PR } & \text { Progesteron Receptor } \\ \text { OCT1 } & \text { Octamer-binding transcription factor } 2 \\ \text { HBBCC } & \text { Henrietta Banting Breast Cancer Collection } \\ \text { FITC } & \text { Fluorescein isothiocyanate } \\ \text { TRITC } & =\text { Tetramethylrhodamine isothiocyanate } \\ \text { DAPI } & =4 \text { ',6-diamidino-2-phenylindole } \\ \text { His } & =\text { Histidine } \\ \text { GST } & =\text { Glutathione S transferase } \\ \text { SP1 } & \text { Specificity Protein 1 } \\ \text { OCT4 } & \text { Octamer-binding transcription factor } 4 \\ \text { AR } & =\end{array}$

\section{REFERENCES}

[1] Desterro JMP, Rodriguez MS, Hay RT. Regulation of transcription factors by protein degradation. Cell Mol Life Sci 2007; 57: 120719.

[2] Zhang Z, Liao B, Xu M, Jin Y. Post-translational modification of POU domain transcription factor Oct-4 by SUMO-1. FASEB J 2007; 21: 3042-51.

[3] Verger A, Perdomo J, Crossley M. Modification with SUMO. A role in transcriptional regulation. EMBO Rep 2003; 4: 137-42.

[4] Nishida T, Terashima M, Fukami K, Yamada Y. PIASy controls ubiquitination-dependent proteasomal degradation of Ets-1. Biochem J 2007; 405: 481-8.

[5] Chen C, Seth, AK, Aplin AE. Genetic and expression aberrations of E3 ubiquitin ligases in human breast cancer. Mol Cancer Res 2006; 4: 695-707.

[6] von Mikecz A. The nuclear ubiquitin-proteasome system. J Cell Sci 2006; 119: 1977-84.

[7] Huang J, Li X, Maguire CA, Hilf R, Bambara RA, Muyan M. Binding of estrogen receptor beta to estrogen response element in situ is independent of estradiol and impaired by its amino terminus. Mol Endocrinol 2005; 19: 2696-712.

[8] Feng Y, Manka D, Wagner KU, Khan SA. Estrogen receptor-alpha expression in the mammary epithelium is required for ductal and alveolar morphogenesis in mice. Proc Natl Acad Sci USA 2007; 104: 14718-23. 
[9] Eakin CM, Maccoss MJ, Finney GL, Klevit RE. Estrogen receptor alpha is a putative substrate for the BRCA1 ubiquitin ligase. Proc Natl Acad Sci USA 2007; 104: 5794-9.

[10] Sentis S, Le Romancer M, Bianchin C, Rostan MC, Corbo L. Sumoylation of the estrogen receptor alpha hinge region regulates its transcriptional activity. Mol Endocrinol 2005; 19: 2671-84.

[11] Burger A, Li H, Zhang XK, et al. Breast cancer genome anatomy: correlation of morphological changes in breast carcinomas with expression of the novel gene product Di12. Oncogene 1998; 16: 327-33.

[12] Burger AM, Gao Y, Amemiya Y, et al. A novel RING-type ubiquitin ligase breast cancer-associated gene 2 correlates with outcome in invasive breast cancer. Cancer Res 2005; 65: 10401-12.

[13] Burger A, Amemiya Y, Kitching R, Seth AK. Novel RING E3 ubiquitin ligases in breast cancer. Neoplasia 2006; 8: 689-95.

[14] Reid G, Hubner MR, Metivier R, et al. Cyclic, proteasomemediated turnover of unliganded and liganded ERalpha on responsive promoters is an integral feature of estrogen signaling. Mol Cell 2003; 11: 695-707.

[15] Lonard DM, Nawaz Z, Smith CL, O'Malley BW. The 26S proteasome is required for estrogen receptor-alpha and coactivator turnover and for efficient estrogen receptor-alpha transactivation. Mol Cell 2000; 5: 939-48.

[16] Wijayaratne AL, McDonnell DP. The human estrogen receptoralpha is a ubiquitinated protein whose stability is affected differentially by agonists, antagonists, and selective estrogen receptor modulators. J Biol Chem 2001; 276: 35684-92.

[17] Fan S, Wang J, Yuan R, et al. BRCA1 inhibition of estrogen receptor signaling in transfected cells. Science 1999; 284: 1354-6.

[18] Saji S, Okumura N, Eguchi H, et al. MDM2 enhances the function of estrogen receptor alpha in human breast cancer cells. Biochem Biophys Res Commun 2001; 281: 259-65.

[19] Nawaz Z, Lonard DM, Smith CL, et al. The Angelman syndromeassociated protein, E6-AP, is a coactivator for the nuclear hormone receptor superfamily. Mol Cell Biol 1999; 19: 1182-9.

[20] Nilsson S, Gustafsson J. Estrogen receptor transcription and transactivation: Basic aspects of estrogen action. Breast Cancer Res 2000; 2: 360-6.

[21] Klinge CM. Estrogen receptor interaction with co-activators and co-repressors. Steroids 2000; 65: 227-51.

[22] Chu I, Arnaout A, Loiseau S, et al. Src promotes estrogendependent estrogen receptor alpha proteolysis in human breast cancer. J Clin Invest 2007; 117: 2205-15.

[23] Reddel RR, Murphy LC, Sutherland RL. Factors affecting the sensitivity of T-47D human breast cancer cells to tamoxifen. Cancer Res 1984; 44: 2398-405.

[24] Feng D, Kan YW. The binding of the ubiquitous transcription factor $\mathrm{Sp} 1$ at the locus control region represses the expression of beta-like globin genes. Proc Natl Acad Sci USA 2005; 102: 9896900.

[25] Karin M, Liu Z, Zandi E. AP-1 function and regulation. Curr Opin Cell Biol 1997; 9: 240-6.

[26] Bing D, Feng-Qi Z. Involvement of the ubiquitous Oct-1 transcription factor in hormonal induction of $\beta$-casein gene expression. Biochem J 2007; 401: 57-64.

[27] Gordon S, Akopyan G, Garban H, Bonavida B. Transcription factor YY1: structure, function, and therapeutic implications in cancer biology. Oncogene 2006; 25: 1125-42.
[28] Helmer EB, Raaka BM, Samuels HH. Hormone-dependent and independent transcriptional activation by thyroid hormone receptors are mediated by different mechanisms. Endocrinology 1996; 137: 390-9.

[29] Xu J, Thompson KL, Shephard LB, Hudson LG, Gill GN. T3 receptor suppression of Sp1-dependent transcription from the epidermal growth factor receptor promoter via overlapping DNAbinding sites. J Biol Chem 1993; 268: 16065-73.

[30] Nakshatri H, Bhat-Nakshatri P, Martin DA, Goulet RJ Jr, Sledge, GW Jr. Constitutive activation of NF-kappaB during progression of breast cancer to hormone-independent growth. Mol Cell Biol 1997; 17: 3629-39.

[31] Garcia-Dominguez M, Reyes JC. SUMO association with repressor complexes, emerging routes for transcriptional control. Biochim Biophys Acta 2009; 1789: 451-9.

[32] Mo YY, Yu Y, Theodosiou E, Ee PL, Beck WT. A role for Ubc9 in tumorigenesis. Oncogene 2005; 24: 2677-83.

[33] Knipscheer $\mathrm{P}$, Flotho A, Klug H, et al. Ubc9 sumoylation regulates SUMO target discrimination. Mol Cell 2008; 31: 371-82.

[34] Kaikkonen S, Jaaskelainen T, Karvonen U, et al. SUMO-specific protease 1 (SENP1) reverses the hormone-augmented SUMOylation of androgen receptor and modulates gene responses in prostate cancer cells. Mol Endocrinol 2009; 23(3): 292-307.

[35] Deng Z, Wan M, Sui G. PIASy-mediated sumoylation of Yin Yang 1 depends on their interaction but not the RING finger. Mol Cell Biol 2007; 27: 3780-92.

[36] Spengler ML, Brattain MG. Sumoylation inhibits cleavage of Sp1 $\mathrm{N}$-terminal negative regulatory domain and inhibits Sp1-dependent transcription. J Biol Chem 2006; 281: 5567-74.

[37] Xu J, Watkins T, Reddy A, Reddy ES, Rao VN. A novel mechanism whereby BRCA $1 / 1 \mathrm{a} / 1 \mathrm{~b}$ fine tunes the dynamic complex interplay between SUMO-dependent/independent activities of Ubc9 on E2-induced ERalpha activation/repression and degradation in breast cancer cells. Int J Oncol 2009; 34: 939-49.

[38] Burger AM, Seth AK. The ubiquitin-mediated protein degradation pathway in cancer: therapeutic implications. Eur J Cancer 2004; 40: 2217-29.

[39] Amemiya Y, Azmi P, Seth A. Autoubiquitination of BCA2 RING E3 ligase regulates its own stability and affects cell migration. Mol Cancer Res 2008; 6: 1385-96.

[40] Connor MK, Azmi PB, Subramaniam V, Li H, Seth A. Molecular characterization of ring finger protein 11. Mol Cancer Res 2005; 3 : 453-61.

[41] Hoeller D, Hecker CM, Dikic I. Ubiquitin and ubiquitin-like proteins in cancer pathogenesis. Nat Rev Cancer 2006; 6: 776-88

[42] Baek SH. A novel link between SUMO modification and cancer metastasis. Cell Cycle 2006; 5: 1492-5.

[43] Qi X, Borowicz S, Pramanik R, Schultz RM, Han J, Chen G. Estrogen receptor inhibits c-Jun-dependent stress-induced cell death by binding and modifying c-Jun activity in human breast cancer cells. J Biol Chem 2004; 279: 6769-77.

[44] Burger AM, Dai F, Schultes CM, et al. The G-quadruplexinteractive molecule BRACO-19 inhibits tumor growth, consistent with telomere targeting and interference with telomerase function. Cancer Res 2005; 65: 1489-96.

This is an open access article licensed under the terms of the Creative Commons Attribution Non-Commercial License (http://creativecommons.org/licenses/by$\mathrm{nc} / 3.0 /$ ), which permits unrestricted, non-commercial use, distribution and reproduction in any medium, provided the work is properly cited. 